\title{
Politainment en campaña: el engagement en la audiencia social de Twitter
}

\author{
Politainment in campaign: engagement on Twitter social \\ audience
}

\author{
Zamora-Medina, R. y Rebolledo-De la Calle, M. ${ }^{1}$ \\ Recibido: 05-04-2021 - Aceptado: 15-08-2021 \\ https://doi.org/10.26441/RC20.2-2021-A20
}

\begin{abstract}
RESUMEN: La presencia de los candidatos en programas de politainment durante las campañas electorales se ha convertido en una práctica generalizada en España. Considerando que la gran mayoría de las reacciones en las redes sociales se refieren al contenido o a los invitados de los programas de televisión (D'heer y Verdegem, 2014), su análisis puede resultar de gran interés para determinar en qué medida estos espectáculos contribuyen a mejorar el conocimiento político de cada candidato a través de los ojos de la audiencia (Boukes y Trillin, 2017).
\end{abstract}

Esta investigación parte de algunos estudios previos que reconocen el impacto de los programas de infoentretenimiento político en la generación de engagement o compromiso político entre la audiencia social (Moy, Xenos y Hess, 2005), y sus beneficios para el acercamiento de nuevos públicos (Teruel, 2016). Sin embargo, pretende dar un paso más, especificando qué tipo de contenidos logran despertar mayor engagement en el público.

Para ello, incluye un análisis empírico comparativo y cuantitativo de la conversación social generada bajo el hashtag oficial designado para cada candidato electoral por parte del programa El Hormiguero durante la campaña electoral de 2019 en España. Concretamente, se analiza el contenido de 249 tuits seleccionados entre aquellos con mayor nivel de interacción entre los prosumidores -métricas de seguimiento, viralidad y temática- tomando como caso de estudio las apariciones públicas de Pablo Iglesias (Unidas Podemos), Pablo Casado (PP), Albert Rivera (Ciudadanos), Santiago Abascal (VOX) e Iñigo Errejón (Más País) Los resultados demuestran la presencia notable de contenidos que no favorecen el conocimiento constructivo de los candidatos, si bien, en función de determinadas variables, pueden favorecer el engagement ciudadano como parte de la estrategia de la campaña.

Palabras clave: politainment; campaña electoral; Twitter; engagement político; encuadre; España.

ABSTRACT: The presence of candidates in politainment programs during electoral campaigns has already become a general practice in Spain. Considering that the vast majority of reactions on social media refer to the content or guests of television programs (D'heer \& Verdegem, 2014), these reactions can be used to determine to what extent these shows contribute to improving the political knowledge of each candidate through the eyes of the audience (Boukes \& Trilling, 2017).

\footnotetext{
${ }^{1}$ Rocío Zamora-Medina es Doctora en Comunicación Pública por la Universidad de Navarra y Profesora Titular en la Facultad de Comunicación de la Universidad de Murcia. Imparte docencia en Comunicación Política y Fundamentos de la Comunicación y de la Información. Sus principales líneas de investigación son innovaciones en Campañas digitales y análisis de procesos de construcción de imagen política en el ecosistema digital. rzamoramedina@um.es, https://orcid.org/0000-0002-0541-2456

Marta Rebolledo-De la Calle es Doctora en Comunicación Política por la Universidad de Navarra y Université Paris-Est. Profesora Contratada Doctor en la Facultad de Comunicación de la Universidad de Navarra donde es directora del Master en Comunicación Política y Corporativa (MCPC). Imparte las asignaturas Political Communication, Campañas Electorales, Contemporary Political Systems. Sus líneas de investigación prioritarias son análisis de campañas electorales y marketing político mrebolledo@unav.es, http://orcid.org/0000-0003-0986-7332
} 
This research is based on some previous studies supporting the impact of political infotainment programs on promoting political engagement among the social audience, (Moy, Xenos \& Hess, 2005) and in terms of benefits for politicians regarding their public image and outreach to new audiences (Teruel, 2016). However, this study aims to go further, specifying what type of content promotes greater public involvement.

For that purpose, we conducted a comparative and quantitative empirical analysis of the social conversation generated from the main hashtag designed for each candidate on the tv show El Hormiguero during the 2019 elections campaign. Concretely, a sample of 249 tweets were analysed, selected among those with a higher level of interaction among prosumers - metrics of follow-up, virality and thematictaking as a case study the public appearances of Pablo Iglesias (Unidas Podemos), Pablo Casado (PP), Albert Rivera (Ciudadanos), Santiago Abascal (VOX) and Íñigo Errejón (Más País) in the program El Hormiguero during the 2019 electoral campaign in Spain. Results show the remarkable presence of contents that do not promote the constructive knowledge of candidates. However, depending on certain variables, this content fosters citizen engagement as part of the campaign strategy.

Keywords: politainment; electoral campaign; Twitter; political engagement; framing; Spain.

\section{Introducción}

El politainment se entiende como un fenómeno directamente conectado con la comunicación política de entretenimiento en cuanto a su producción, difusión y consumo en diferentes formatos $(\mathrm{Be}-$ rrocal, 2017). El alcance y repercusión pública va más allá de la mera exposición pública y “celebrificación" de los políticos (Oliva, Pérez-Latorre y Besalú, 2015) en la televisión, abarcando también los espacios digitales, donde tienen lugar las interacciones entre los programas de politainment y su audiencia social. Es precisamente en estos escenarios donde puede apreciarse la repercusión real de la actuación de los líderes políticos, a partir de la conversación social generada, en cada caso, con una audiencia social activa, conectada y empoderada (Alonso-Muñoz y Casero-Ripollés, 2017; Marinelli y Andò, 2016; Aroldi y Vittadini, 2015; Giglietto y Selva, 2014; Echegaray y Peñafiel, 2013).

El politainment conlleva diferentes consecuencias con respecto a las dinámicas de comunicación, como es la devaluación informativa política, junto con la pérdida de la calidad democrática (Sayre y King, 2010; Schultz, 2012), dando protagonismo a un entorno comunicativo de la posverdad y a la "celebrización de políticos" en los géneros de entretenimiento (Oliva et al., 2015).

Se trata de un fenómeno reciente relativo a la política espectacularizada basada en información sobre política y entretenimiento, funciones relacionadas con los medios (Nieland, 2008; Sayre y King, 2010; Schultz, 2012). También este término aparece conceptualizado como el "ablandamiento" de la comunicación política (Otto, Glogger y Boukes, 2016) que surgió en los años 90 con la intensa competencia en el mercado audiovisual, abarcando todo tipo de temas, incluidos aquellos pertenecientes al ámbito político. A partir de ese momento, este fenómeno ha adquirido un sólido corpus de estudios para ser considerado un tema de investigación en comunicación política (Berrocal, 2017).

Si bien la mayoría de los estudios sobre politainment se basan en la televisión, en el contexto de la sociedad digital, las redes sociales se convierten en plataformas clave para estudiar esta tendencia periodística hacia la representación de la realidad como actuación o espectáculo (Tyron, 2008; Towner y Dulio, 2011; Berrocal, Campos-Domínguez y Redondo, 2012). En el contexto de politainment, las redes sociales juegan un papel importante como instrumentos para el intercambio de información, tanto horizontal como verticalmente, desde una audiencia social activa, conectada y empoderada caracterizada por participar, compartir, producir y colaborar (Alonso-Muñoz y Casero-Ripollés, 2017; Marinelli y Andò, 2016; Aroldi y Vittadini, 2015; Echegaray y Peñafiel, 2013). Estas interacciones son clave para los programas de televisión con el fin de completar su significado 
a partir de contenidos difundidos en las redes sociales y para facilitar la participación del público (Guo y Chan-Olmsted, 2015; Moy et al., 2005).

En este sentido, también existen estudios relevantes sobre el uso del infoentretenimiento político en Twitter (Berrocal, Zamora-Medina y Rebolledo, 2021; Baviera, Peris y Cano-Orón, 2019; López-Meri, Marcos-García y Casero-Ripollés, 2017), o Facebook (Durántez-Stolle y MartínezSanz, 2019). Recientemente, algunos estudios también se han centrado en la participación colectiva relacionada con el uso de Youtube (Berrocal, Campos-Domínguez, Redondo-García, 2012) o incluso el papel de los prosumidores en Youtube (Berrocal, Campos-Domínguez y Redondo, 2014; Berrocal, Gil Torres; Campos-Domínguez, 2016).

La presente investigación representa una aproximación al estudio de la audiencia social del politainment y la generación de engagement político en las conversaciones de Twitter relacionadas con los programas de entretenimiento político en España. Entender la audiencia social es clave para comprender el impacto real de este fenómeno. Las interacciones que se establecen en ella completan el significado del contenido difundido en las redes sociales y por parte de los programas politainment, según algunos estudios, puede facilitar la participación de la audiencia (Moy et al., 2005; Guo y Chan-Olmsted, 2015).

Dado el estado actual de los estudios citados, el principal objetivo de esta investigación será investigar qué tipo de contenidos vinculados con los programas de entretenimiento político generan más engagement en la audiencia social, así como mejorar el conocimiento de la conversación social de Twitter y su repercusión sobre la formación de conocimiento político en la ciudadanía.

Partimos de la hipótesis de que este tipo de programas con contenido relacionado con la espectacularización de la política (noticias suaves, parodia, tratamiento superficial, entre otros aspectos) implica un grado de engagement mayor que en el que se consigue bajo fórmulas tradicionales en las que se difunden contenidos políticos. Sin embargo, en la medida en la que se intensifican algunos encuadres específicos, vinculados con los recursos emocionales y la personalización, incrementará el nivel de implicación por parte de la ciudadanía.

Por todo ello, nos proponemos las siguientes preguntas de investigación:

RQ1.: ¿Qué diferencias o similitudes se identifican, desde un punto descriptivo o formal, entre los tuits que se originaron en torno a las apariciones de los distintos candidatos que acudieron al programa El Hormiguero?

RQ2.: ¿En qué medida los contenidos difundidos a través de Twitter durante o tras la emisión de un programa de entretenimiento político como El Hormiguero incluyen recursos para facilitar el conocimiento político constructivo en la audiencia social?

RQ3.: ¿Qué tipo de contenidos vinculados con la conversación social sobre los mencionados programas de politainment logran despertar mayor engagement en el público?

\section{Marco teórico}

\subsection{La audiencia social como elemento clave en los programas de politainment}

En un entorno mediático caracterizado por la multiplicación y competencia de los medios, estos se enfrentan a la fragmentación de la audiencia mediante contenidos que demandan la atención de los usuarios, siendo accesibles, baratos y populares (Berrocal et al., 2012; Ortells, 2011). Al mismo tiempo, en la era de la post-televisión, la espectacularización ha aumentado y la interactividad del contenido se convierte en el principal valor para la audiencia. En este contexto se sitúan los pro- 
gramas de politainment: comparten contenidos relacionados con la política espectacularizada o la política transformada en entretenimiento (Berrocal, 2017). De hecho, el término alude al "enredo de actores políticos, temas y procesos con la cultura del entretenimiento" (Nieland, 2008).

Teniendo como base de fondo la importancia de la información versus el entretenimiento, existen diferentes formatos de politainment que pueden organizarse en tres categorías (Jiménez, Barrio y Guinovart, 2017): programa político espectacularizado, programa de entretenimiento con contenido político (programa no informativo) e infoshow (satírico, parodia, humor). En un extremo, incluimos aquellos programas que incluyen más información política y menos entretenimiento en comparación con otros géneros, al igual que el programa político espectacularizado. En el extremo opuesto, consideramos aquellos programas con menos información política, pero con un enfoque más de entretenimiento, como los infoshows. En el medio, situamos los programas de entretenimiento con contenido político, en los que se incluyen magazines políticos y contenidos similares con un alto grado de entretenimiento, pero menos que en la parodia.

Las interacciones que suscitan estos formatos televisivos constituyen la última pieza que componen éstos ya que completarán su significado a través de las redes sociales. De hecho, las reacciones de la audiencia revelan las cuestiones que realmente provoca la atención de los espectadores del programa, y proporcionan, a su vez, una visión más profunda en torno a los posibles efectos que pueden generar contenidos que han sido enmarcados bajo diferentes encuadres o frames. Sobre la base conceptual apuntada por la teoría del encuadre o framing, partimos de la idea de que los énfasis realizados en las narrativas informativas pueden tener una influencia notable en la manera como los sujetos perciben aspectos determinados del relato noticioso (Entman, 1993). De tal modo que los propios tuits también pueden contener contenidos que siguen algunos de los marcos o frames apuntados en la literatura, tales como el conflicto, las consecuencias económicas, el interés humano, la moralidad y la atribución de responsabilidad (Semetko y Valkenburg, 2000).

Por otra parte, la respuesta en redes sociales mientras se ve un programa ha llamado la atención entre los académicos; se han acuñado términos como "televisión social” (Buschow, Schneider y Ueberheide, 2014) y "co-visualización remota" (Boukes y Trilling, 2017). Asimismo, cada vez más común es el concepto de "uso de segunda pantalla" o "consumo de doble pantalla" (Giglietto y Selva, 2014) para referirse a la acción de comentar y compartir en redes mientras los usuarios consumen contenido en televisión. Esta realidad puede entenderse como un indicador de participación con la televisión (Pittman y Tefertiller, 2015) o incluso como una forma de "anotación comunitaria" de programas de televisión al reflejarse en los tuits tanto la estructura como el contenido del programa (Diakopoulos y Shamma, 2010; Shamma, Kennedy y Churchill, 2009). De esta manera, los medios promueven esta práctica activamente para mejorar el engagement y lealtad de la audiencia. Debido a que la gran mayoría de las reacciones en redes sociales se refieren al contenido o a los invitados de los programas de televisión política (D'heer y Verdegem, 2014), estas reacciones constituyen un valor para determinar la sustantividad política de estos programas a través de los ojos de la audiencia (Boukes y Trilling, 2017).

\subsection{El engagement digital en los programas de infoentretenimiento político}

Desde una perspectiva académica, el engagement se considera una cuestión de investigación multidisciplinar de notable actualidad para analizar el impacto de las redes sociales, que precisa de más aclaraciones conceptuales (Chang-Olmsted, Wolter y Wang, 2017; Macnamara, Sakinofsky y Beattie, 2012). De hecho, entre los diferentes significados de engagement, este concepto se entiende como un proceso de interacción entre algunos actores — ciudadanos, usuarios, clientes, etc. - y algunas organizaciones —administración pública, empresas, etc.— (Ballesteros, 2019). De tal modo que, grosso modo, el engagement político se ha equiparado con la participación política, concretada en algunos comportamientos como las intenciones de voto o la discusión política interpersonal. Sin 
embargo, también tiene que ver con la participación cívica, que implica la comunicación ciudadana horizontal para lograr mejoras en sus comunidades y construir capital social.

En el contexto de la digitalización, la investigación sobre este asunto está vinculada al "engagement de las redes sociales" (Paine, 2011), el "engagement online" (Cvijikj y Michahelles, 2013) o el "engagement digital" (Valerio, Herrera-Murillo, Villanueva-Puente, Herrera-Murillo y Rodríguez, 2015). La mayoría de estos estudios se refieren al engagement y a una mayor participación de los usuarios en las redes sociales como consecuencia de las publicaciones compartidas por otros usuarios. Respecto al vínculo entre redes sociales y engagement digital, una de las suposiciones exploradas con mayor frecuencia es que los medios digitales tienen la capacidad de permitir a los ciudadanos interactuar entre ellos, pasar tiempo navegando a través de una gran cantidad de información y exponerse a problemas de muy diversa naturaleza, llevándolos eventualmente incluso a tomar medidas cívicas o políticas (Boulianne y Theocharis, 2018). Se entiende, por tanto, que los medios digitales pueden desempeñar el papel de espacios online en los que los ciudadanos pueden obtener las habilidades necesarias para participar en la vida cívica (Kahne y Bowyer, 2018; Chae, Lee y Kim, 2019; Skoric, Zhu, Goh y Pang, 2016; Boulianne, 2009) al considerar que las interacciones sociales con personas y valores diferentes pueden tener un efecto movilizador.

En el contexto del entretenimiento político, donde la información política se ofrece bajo los formatos políticos espectacularizados, la importancia de las redes sociales en la construcción de comunidades online que conducirían a una exposición incidental tiene una mayor repercusión política (Kahne y Bowyer, 2018). Este tipo de contenidos, caracterizados por noticias blandas, podrían reducir la desafección por la política y lograr el acercamiento de determinados públicos; sin embargo, no necesariamente supondría una mejora del conocimiento político real a largo plazo (Baum, 2003). En cualquier caso, el impacto directo de los formatos de politainment en la participación de los ciudadanos no es generalizable y requiere de más análisis. Así lo demostraron Moy et al. (2005) en su estudio sobre los efectos políticos del infoentretenimiento, al observar que el infotainment político puede mejorar el engagement político, pero no para todos los sectores del electorado y no durante todo el tiempo.

\section{Metodología}

La selección muestral de la investigación toma como caso de estudio las apariciones de cada uno de los candidatos electorales en el programa El Hormiguero durante la campaña electoral de las elecciones generales celebradas en España el 10 de noviembre de 2019. Este espacio televisivo está producido por la compañía 7 y acción y se emite en la cadena generalista Antena 3 desde el 5 de septiembre de 2011 (anteriormente en Cuatro de 2006 al 2011) en la franja horaria del prime time.

Dentro de la clasificación de programas politainment, El Hormiguero se corresponde con la categoría de programa de entretenimiento con contenido político; esto es, se trataría de un programa no informativo. La elección de este programa se debe a que se trata de uno de los programas más representativos de dicha categoría, goza de gran popularidad debido a su larga permanencia en la parrilla televisiva ${ }^{2}$. Además, es el único en el que se dispone de emisiones con presencia de prácticamente todos los candidatos a la presidencia del Gobierno.

El programa modificó la estructura habitual de su contenido — que gira en torno al humor, los experimentos o la magia con la participación activa de distintos colaboradores fijos- para emitir una serie de entrevistas de contenido político a los candidatos Santiago Abascal (VOX), Íñigo Errejón (Más

\footnotetext{
${ }^{2}$ El programa en el que apareció el candidato Santiago Abascal fue uno de los cincuenta eventos más vistos en televisión en el año 2019, con un 23,5\% de share (Barlovento, 2019). Para más información: https://www.barloventocomunicacion.es/audiencias-anuales/analisis-televisivo-2019
} 
País), Pablo Casado (PP), Albert Rivera (Ciudadanos) y Pablo Iglesias (Unidas Podemos). Tal y como muestra la tabla 1, todos los principales candidatos fueron incluidos, a excepción del aspirante del Partido Socialista Obrero Español (PSOE) Pedro Sánchez, quien decidió no asistir al programa,

Tabla 1. Orden cronológico de la aparición de los candidatos a El Hormiguero

\begin{tabular}{|c|c|c|c|c|}
\hline Candidato & Fecha emisión & Hashtag oficial & $\begin{array}{c}\text { Audiencia } \\
\text { televisión }\end{array}$ & Share (\%) \\
\hline Santiago Abascal & $10 / 10 / 2019$ & \#SantiagoAbascalEH & 4.049 .000 & $23,5 \%$ \\
\hline Íñigo Errejón & $15 / 10 / 2019$ & \#ErrejónEH & 2.089 .000 & $11,5 \%$ \\
\hline Pablo Casado & $24 / 10 / 2019$ & \#PabloCasadoEH & 2.049 .000 & $11,7 \%$ \\
\hline Albert Rivera & $28 / 10 / 2019$ & \#AlbertRiveraEH & 2.458 .000 & $14,3 \%$ \\
\hline Pablo Iglesias & $31 / 10 / 2019$ & \#PablolglesiasEH & 1.477 .000 & $10,4 \%$ \\
\hline
\end{tabular}

Fuente: Elaboración propia y Kantar Media.

$\mathrm{Al}$ tratarse de una primera aproximación al estudio del politainment y su relación con la generación del engagement digital, se optó por un diseño metodológico que comprendía el análisis cuantitativo aplicado al contenido de una muestra de 249 tuits $^{3}$ con mayor nivel de interacción entre los prosumidores. En él se incluía los 50 principales tuits correspondientes a cada una de las apariciones de los candidatos bajo el hashtag oficial designado para cada emisión por parte del programa El Hormiguero. Se trata de los mensajes que más likes generaron durante la jornada de emisión del espacio televisivo. Consideramos que la variable likes representa una categoría adecuada para establecer esta clasificación al resultar una reacción instantánea y natural por parte de los usuarios de la red social Twitter. Además, se evaluaron y clasificaron en categorías grupales el número de comentarios y retuits concebidos por cada uno de estos tuits seleccionados.

El proceso de codificación fue realizado manualmente por los tres autores de la investigación a través de una ronda de análisis de una muestra común del 10\% de la muestra (10 tuits de cada uno de los candidatos hasta completar 50 tuits) con la que se aseguró una verificación de confiabilidad intercodificadores de .91 en todas las categorías, utilizando la fórmula de Holsti.

Se realizó un análisis empírico comparativo y cuantitativo del contenido emitido y se registró asimismo el nivel de interacción entre los prosumidores en lo referente a seguimiento, viralidad y temática.

Entre las principales variables se incluyeron aspectos como la existencia de enlaces a contenidos, mención de perfiles, uso de emoticonos y elementos audiovisuales, así como otras características del fenómeno del politainment en los contextos políticos como el tipo, tono y relevancia de la información aportada, el encuadre y tipo de lenguaje del tuit y la intencionalidad directa o indirecta de los distintos sujetos emisores del mensaje mediante tablas de contingencia para identificar posibles asociaciones entre las variables incluidas en el estudio.

Para medir empíricamente el engagement digital, diferentes trabajos han utilizado métodos cuantitativos y cualitativos; a veces incluso se combinan con el uso del índice de participación (Ballesteros, 2017; Muñiz y Ballesteros, 2016; Oviedo-García, Muñoz-Expósito, Castellanos-Verdugo y Sánchez-Mejías, 2014) en función de diferentes variables de participación (likes, comentarios, compartir, etc.). En este estudio, medimos el engagement a partir de la elaboración de un índice, en función del cómputo en la adición de las variables de registro y alcance: likes, comentarios y retuits

\footnotetext{
${ }^{3}$ La muestra ha sufrido una variación al eliminarse la cuenta oficial de uno de los mensajes escogidos en la aparición del candidato Santiago Abascal.
} 
de cada uno de los tuits publicados. De este modo, se identificaron tres niveles numéricos de engagement (bajo, medio y alto). Dicha variable resultante se cruzó con las diferentes categorías de la hoja de análisis (aspectos formales, tema principal, encuadre, lenguaje, intencionalidad) y se utilizó el estadístico Chi-cuadrado de Pearson $(\leq .05)$ para determinar el nivel de significación estadística.

El análisis estadístico se llevó a cabo utilizando el programa informático SPSS versión 24.

\section{Resultados}

\subsection{Aspectos formales y descriptivos de los tuits}

Desde un punto descriptivo, el análisis de las frecuencias simples nos permite observar diferencias interesantes entre el tipo de tuits que se originan en torno a las apariciones de los distintos candidatos que acudieron al programa El Hormiguero respecto a las variables analizadas.

Partimos del hecho de que, en líneas generales, la intervención de Abascal fue la que suscitó mayor viralidad (casi un $82 \%$ de sus tuits obtuvieron más de 50 comentarios; similar, de igual modo, al número de retuits y el número de likes) en comparación con el resto de los candidatos.

Tal y como señalamos en la RQ1, nos preguntamos qué diferencias o similitudes se identifican, desde un punto descriptivo o formal, entre los tuits que se originaron en torno a las apariciones de los distintos candidatos que acudieron al programa El Hormiguero.

En ese sentido, por lo que se refiere a las cuestiones formales, los tuits generados por la audiencia social no incluían enlace en su mayoría (54,2\%), y sólo en uno de cada cuatro casos enlazaba a un video del programa. Sólo los tuits de Casado (48\%) y sobre todo de Rivera (62\%) enlazaron a contenidos externos, tales como videos del programa en el primer caso y otros medios o webs diferentes, en el segundo. Además, buena parte de los tuits no incluyeron ninguna mención $(42,6 \%)$, y cuando lo hacían se referían únicamente a los propios candidatos invitados al programa $(49,8 \%)$. Casado fue el político que contó con más menciones propias, mientras que Errejón fue el menos mencionado de todos.

La inclusión de emoticonos en los tuits sigue sin ser un recurso generalizado por parte de la audiencia social de los programas de politainment. En los pocos casos que son incluidos suelen indicar alegría. Otro dato significativo es que un buen número de tuis $(29,7 \%)$ no incluían ningún tipo de contenido audiovisual. Cuando sí lo hacían optaron por el vídeo (37,8\%)- más evidente entre los tuits de Casado- y, en menor medida, capturas de pantalla procedente de medios (13,3\%) -en especial en el caso de Rivera- o fotografías (13,3\%). El protagonista de estos elementos audiovisuales era siempre el político invitado al programa.

En lo que se refiere al tipo de contenidos que se difundieron, destacan los mensajes transmitidos desde el estilo soft news $(81,5 \%)$ frente a las hard news $(18,5)$. Este dato indica que se opta por un enfoque mayoritariamente superficial, haciendo hincapié en detalles, la parte humana y en aspectos concretos, en lugar de establecer conexiones con otras noticias o temas amplios y de calado. Si bien se trata de un comportamiento similar en todos los candidatos, lo cierto es que la conversación en torno a Errejón y a Rivera incluyó un porcentaje mayor de hard news que el resto de los candidatos ( $26 \%$ en cada caso), en forma de comentarios basados en análisis e información factual, apoyados en datos, y cuya información es relevante y de interés.

Como dato a tener en cuenta destacamos que la conversación social estuvo claramente personificada en la figura de los candidatos $(55,4 \%)$ frente a la de los partidos $(15,3 \%)$ o las políticas públicas (26,9\%). Esta personificación resultó más evidente en el caso de Errejón (86\%) o Abascal $(65,3 \%)$ que en el resto de los candidatos. Sólo tras la intervención de Casado, la conversación social incluyó, además, un número considerable de menciones al partido político. 
La conversación social se centró, en buena medida, en los políticos y la clase política como cuestión o tema público prioritario $(49,8 \%)$, y estuvo enmarcada a través de un relato coloquial $(69,9 \%)$ definido por un tono positivo y negativo en términos similares. Se apreciaron diferencias significativas entre los diferentes candidatos, ya que dicha conversación fue considerablemente más positiva que negativa en el caso de Abascal (40\%) y de Rivera (56\%), mientras que ocurrió lo contrario en el caso de la conversación social a raíz de la intervención de Iglesias (con un $72 \%$ de negatividad) y en menor medida en el caso de Errejón (46\%). Otro asunto relevante de la conversación fueron los problemas de desigualdad social (12,9\%), sobre todo en el caso de Iglesias (28\%) y, curiosamente, también Abascal (18,4\%).

Respecto a la intencionalidad de los tuits difundidos, destacaron las conversaciones que incluían una intencionalidad estrictamente informativa (30,5\%) y aquellas que buscaban la adhesión social $(28,5 \%)$ o la crítica $(26,5 \%)$. En este sentido, se aprecian también algunas diferencias entre los candidatos, teniendo en cuenta que la mitad de los casos vinculados a Iglesias tuvieron una clara intención crítica, mientras que la conversación sobre Casado tuvo un componente claramente favorable a la adhesión social (40\%).

\subsection{Relevancia del contenido}

Una de las variables claves de nuestra investigación fue identificar el tipo de contenido que ofrecen los tuits sobre politainment en los contextos políticos. Así, en la RQ2 nos preguntamos en qué medida los contenidos difundidos a través de Twitter durante o tras la emisión de un programa de entretenimiento político como El Hormiguero incluyen recursos para facilitar el conocimiento político constructivo en la audiencia social.

En general, destacaron los tuits caracterizados por un contenido político vacío (58\%) frente a los que mostraron contenido político constuctivo (30\%) o contenido no político (22\%). Este dato demuestra que los tuits emitidos tras la emisión de estos programas no ofrecieron contenidos que facilitaran el conocimiento político entre la audiencia social.

Uno de los cruces que resultó estadísticamente significativo fue la relación entre la relevancia del contenido y la intencionalidad de los tuits (Chi-square $\leq .000$ ). Tal y como se aprecia en la tabla 2 , aquellos tuits que ofrecieron un contenido político vacío o carente de interés, en lugar de buscar la difusión de información, se caracterizaron por estar enmarcados en una intencionalidad de búsqueda de adhesión (37,7\%) o bien buscaban la expresión de críticas (29\%). Por el contrario, los tuits que realmente aportaban contenido político constructivo claramente sí que optaron por la intencionalidad informativa $(67,3 \%)$. Entre ambas opciones se situaron los tuits de contenido no político, que presentaron una intencionalidad entre crítica $(34,4 \%)$ e informativa $(31,1 \%)$.

Tabla 2. Relación entre la relevancia del contenido y la intencionalidad de los tuits

\begin{tabular}{|l|l|l|l|l|l|l|}
\hline & Informativo & Jocoso & Crítico & Adhesión & Otros & Total \\
\hline Contenido político constructivo & $67,3 \%$ & $0,0 \%$ & $10,2 \%$ & $22,4 \%$ & $0,0 \%$ & $100,0 \%$ \\
\hline Contenido político vacío & $17,4 \%$ & $15,2 \%$ & $29,0 \%$ & $37,7 \%$ & $0,7 \%$ & $100,0 \%$ \\
\hline Contenido no político & $31,1 \%$ & $19,7 \%$ & $34,4 \%$ & $13,1 \%$ & $1,6 \%$ & $100,0 \%$ \\
\hline Total & $30,5 \%$ & $13,3 \%$ & $26,5 \%$ & $28,5 \%$ & $1,2 \%$ & $100,0 \%$ \\
\hline
\end{tabular}

Fuente: Elaboración propia.

También se detectó una relación estadísticamente significativa entre la relevancia del contenido y el tono de los tuits (Chi-square $\leq .054)$ : los mensajes que favorecían el conocimiento político fueron también los que menor presencia de negatividad presentaban. De tal modo que el tono positivo estu- 
vo presente mayoritariamente entre los tuits de contenido político constructivo $(57,1 \%)$ y, en menor medida, en los de contenido político vacío (44,2\%). Por su parte, el tono negativo sólo caracterizó a éstos últimos, así como a aquellos tuits de contenido no político.

Otro cruce estadísticamente significativo fue la relación entre la relevancia del contenido y el encuadre de los tuits (Chi-square $\leq .002)$. Los tuits de contenido político constructivo utilizaron mayoritariamente el marco de la conflictividad (51\%), un dato no tan evidente en el caso de aquellos tuits de contenido político vacío, para los que la conflictividad (35,5\%) se alternó con la emotividad o interés humano $(21,7 \%)$ y la moralidad $(20,3 \%)$. Los tuits de contenido no político se enmarcaron claramente sobre la emotividad o interés humano $(31,1 \%)$.

En cuanto a la relación entre el tipo de contenido y el tipo de información que se trate, en función de si puede ser considerada una información blanda (soft news) o información dura (hard news), también pudo observarse una relación significativa entre estas variables (Chi-square $\leq .054)$. Como era de esperar, los contenidos políticos constructivos se asociaban claramente con noticias duras $(81,6 \%)$, mientras que los contenidos políticos vacíos se asociaron, por el contrario, con noticias blandas $(97,1 \%)$. Algo similar se observó con los contenidos no políticos, al vincularse en su mayoría con noticias blandas $(96,7 \%)$.

También pudo comprobarse la relación entre la relevancia del contenido y el protagonista del contenido que aparecía en cada tuit (Chi-square $\leq .001)$. Así, en aquellos contenidos políticamente constructivos solían tener como protagonista un tema o política pública (49\%) y, en menor medida, a los propios candidatos $(36,7 \%)$. Por el contrario, cuando se trataba de tuits caracterizados por un contenido vacío, observamos que el mayor porcentaje correspondía con la figura del candidato (58\% de los casos), un hecho que se repite para los tuits de contenido no político $(73,8 \%$ ).

Finalmente, el análisis de estas asociaciones entre los diferentes tipos de contenido se completa con su vinculación con los temas de los que tratan los tuits, un cruce que también resultó estadísticamente significativo (Chi-square $\leq .000$ ). Como se refleja en la tabla 3 , los tuits de contenido político vacío se concentraron en temas vinculados con la clase política y los políticos en buena medida $(60,9 \%)$. Aquellos contenidos políticos de naturaleza constructiva, si bien diversificaron más su temática, también se caracterizaron con temas vinculados con la clase política $(36,7 \%)$.

Tabla 3. Relación entre el tipo de contenido y tipo de información

\begin{tabular}{|l|c|c|c|c|c|c|} 
& $\begin{array}{c}\text { Cuestión } \\
\text { territorial }\end{array}$ & $\begin{array}{c}\text { Problemas } \\
\text { económicos }\end{array}$ & $\begin{array}{c}\text { Desigualdad } \\
\text { social }\end{array}$ & Inmigración & $\begin{array}{c}\text { Políticos y } \\
\text { clase política }\end{array}$ & Otros \\
\hline $\begin{array}{l}\text { Contenido político } \\
\text { constructivo }\end{array}$ & $14,3 \%$ & $14,3 \%$ & $16,3 \%$ & $4,1 \%$ & $36,7 \%$ & $14,3 \%$ \\
\hline Contenido político vacío & $8,0 \%$ & $8,0 \%$ & $4,3 \%$ & $0,0 \%$ & $60,9 \%$ & $18,8 \%$ \\
\hline Contenido no político & $0,0 \%$ & $1,6 \%$ & $29,5 \%$ & $0,0 \%$ & $36,1 \%$ & $32,8 \%$ \\
\hline Total & $7,2 \%$ & $7,6 \%$ & $12,9 \%$ & $0,8 \%$ & $49,8 \%$ & $21,7 \%$ \\
\hline
\end{tabular}

Fuente: Elaboración propia

\subsection{Politainment y nivel de engagement}

Por último, otro de los objetivos de este estudio era determinar en qué medida los programas de politainment contribuyen a mejorar el engagement político con la audiencia, concretamente, en forma de respuesta social a dichos contenidos. Concretamente, nos preguntamos en la RQ3 qué tipo de contenidos vinculados con la conversación social sobre los mencionados programas de politainment lograron despertar mayor engagement en el público. 
Con tal fin, se realizaron tablas de contingencia entre diferentes variables a partir de las cuales se pudieran identificar ciertas relaciones significativas vinculadas con el mayor o menor nivel de engagement.

Para ello, se consideró como referencia la variable categórica que incluía los tres niveles engagement (bajo, medio, alto) y que, como se ha explicado, tomaba como base el número de retuits, el número de likes y el número de comentarios. Dicha variable se cruzó con otros factores categóricos incluidos en nuestra hoja de análisis, como el protagonista del contenido, el tema principal, el tipo de encuadre, el tipo de información, la relevancia del contenido, el tono, el tipo de lenguaje y la intencionalidad del tuit. Se utilizó el estadístico Chi-cuadrado de Pearson $(\leq .05)$ para comprobar el nivel de significación estadística que, finalmente, sólo se pudo comprobar en el caso de las tres primeras variables mencionadas.

Como se observa en la tabla 4, se identificó una primera relación estadísticamente significativa entre el nivel de engagement de cada tuit y el protagonista del contenido (Chi-square $\leq .054)$ : cuando el candidato era el protagonista, el nivel de engagement del tuit fue considerablemente superior, si se compara con los casos en los que el protagonista era el partido. En los casos en los que el protagonismo era un issue o política pública, el nivel de engagement fue inesperadamente alto, aunque siempre inferior a cuando se trataba de un candidato.

Tabla 4. Relación entre el nivel de engagement y el protagonista del contenido

\begin{tabular}{|l|c|c|c|c|} 
& $\begin{array}{c}\text { Nivel bajo } \\
\text { engagement }\end{array}$ & $\begin{array}{c}\text { Nivel medio } \\
\text { engagement }\end{array}$ & $\begin{array}{c}\text { Nivel alto de } \\
\text { engagement }\end{array}$ & Total \\
\hline Candidato & $29,8 \%$ & $34,8 \%$ & $35,4 \%$ & $100,0 \%$ \\
\hline Partido político & $44,7 \%$ & $34,2 \%$ & $21,1 \%$ & $100,0 \%$ \\
\hline Issue/ política & $23,9 \%$ & $28,4 \%$ & $47,8 \%$ & $100,0 \%$ \\
\hline Total & $30,5 \%$ & $32,9 \%$ & $36,5 \%$ & $100,0 \%$ \\
\hline
\end{tabular}

Fuente: Elaboración propia.

Asimismo, se comprobó la relación entre el nivel de engagement y el tema principal (Chi-square $\leq .013$ ). Algunos temas como los problemas de desigualdad social o la inmigración obtuvieron niveles de engagement considerablemente altos. También resultó alto el engagement de los tuits sobre la cuestión territorial de España, aunque en este caso el patrón no fue tan claro. Los tuits sobre los políticos y la clase política, por su parte, si bien fueron los más numerosos, se circunscribieron en un $70 \%$ de los casos a un nivel de engagement medio o bajo, como se observa en la tabla 5.

Tabla 5. Relación entre el nivel de engagement y el tema principal

\begin{tabular}{|l|c|c|c|c|}
\hline Cuestión territorial España & $\begin{array}{c}\text { Nivel bajo } \\
\text { engagement }\end{array}$ & $\begin{array}{c}\text { Nivel medio } \\
\text { engagement }\end{array}$ & $\begin{array}{c}\text { Nivel alto de } \\
\text { engagement }\end{array}$ & \begin{tabular}{c} 
Total \\
\hline Problemas económicos/paro
\end{tabular} \\
\hline $44,4 \%$ & $11,1 \%$ & $44,4 \%$ & $100,0 \%$ \\
\hline Problemas desigualdad social & $12,5 \%$ & $52,6 \%$ & $26,3 \%$ & $100,0 \%$ \\
\hline Inmigración & $0,0 \%$ & $28,1 \%$ & $59,4 \%$ & $100,0 \%$ \\
\hline Políticos y clase política & $35,5 \%$ & $3,0 \%$ & $100,0 \%$ & $100,0 \%$ \\
\hline Otros & $29,6 \%$ & $31,5 \%$ & $38,9 \%$ & $100,0 \%$ \\
\hline Total & $30,5 \%$ & $32,9 \%$ & $36,5 \%$ & $100,0 \%$ \\
\hline
\end{tabular}

Fuente: Elaboración propia 
Finalmente, tal y como se preveía, se detectó una relación estadísticamente significativa entre el nivel de engagement y el encuadre del tuit (Chi-square $\leq .005$ ). Como muestra la tabla 6, los tuits que utilizaron el encuadre de la emotividad fueron los que puntuaron más alto en nivel de engagement (más de la mitad de este grupo) dejando claro la importancia que tienen los recursos emocionales para generar mayor adhesión en la audiencia. El enfoque del conflicto y del humor como marcos interpretativos también alcanzaron los máximos niveles de engagement. Por el contrario, aquellos tuits bajo el enfoque de la moralidad o de atribución de responsabilidad presentaron niveles de engagement considerablemente más bajos.

Tabla 6. Relación entre el nivel de engagement y el marco del tuit

\begin{tabular}{|l|l|l|l|l|}
\hline No aplicable & $\begin{array}{c}\text { Nivel bajo } \\
\text { engagement }\end{array}$ & $\begin{array}{c}\text { Nivel medio } \\
\text { engagement }\end{array}$ & $\begin{array}{c}\text { Nivel alto de } \\
\text { engagement }\end{array}$ & \multicolumn{1}{|c|}{ Total } \\
\hline Moralidad & $19,4 \%$ & $48,4 \%$ & $32,3 \%$ & $100,0 \%$ \\
\hline Conflictividad & $51,1 \%$ & $31,1 \%$ & $17,8 \%$ & $100,0 \%$ \\
\hline Emotividad/interés humano/dramatización & $24,1 \%$ & $34,9 \%$ & $41,0 \%$ & $100,0 \%$ \\
\hline Atribución de responsabilidad & $25,9 \%$ & $22,2 \%$ & $51,9 \%$ & $100,0 \%$ \\
\hline Humor & $47,1 \%$ & $35,3 \%$ & $17,6 \%$ & $100,0 \%$ \\
\hline Total & $26,3 \%$ & $31,6 \%$ & $42,1 \%$ & $100,0 \%$ \\
\hline
\end{tabular}

Fuente: Elaboración propia.

\section{Discusión}

El análisis empírico de la conversación social generada a partir de las apariciones públicas de cinco líderes políticos -Pablo Iglesias (Unidas Podemos), Pablo Casado (PP), Albert Rivera (Ciudadanos), Santiago Abascal (VOX) e Iñigo Errejón (Más País) - en el mismo programa de infoentretenimiento político, El Hormiguero, durante la campaña electoral de 2019 en España nos ha permitido identificar algunos resultados interesantes al respecto.

En general, nuestros datos demuestran que la mayoría de los tuits que se refieren a los candidatos tras su aparición en dicho programa presentaban un contenido político vacío, es decir, no favorecen el conocimiento constructivo del candidato. Por el contrario, sólo comentaban asuntos desde una visión superficial, mediante una conversación social extremadamente coloquial, centrada mayoritariamente en la figura de los políticos y de la clase política, frente a otros temas concretos. Este dato se confirma con la prevalencia de mensajes codificados como noticias blandas o soft news frente a los tuits encuadrados dentro de las noticias duras o hard news, que caracterizaron los escasos mensajes reconocidos como contenidos políticos constructivos. En otro estudio similar sobre la conversación social en Twitter, Berrocal, Zamora-Medina y Rebolledo (2021), aparte de hacerse eco de la escasa interacción de la audiencia, también señalaban la preponderancia de tuits considerados como soft news.

De hecho, resulta de especial interés la estrecha relación detectada entre aquellos tuits con contenido político irrelevante y la intencionalidad del tuit: los primeros mayoritariamente buscaban la simple adhesión al candidato, mientras que los mensajes con contenido político más constructivo se caracterizaron por tener como prioridad la intencionalidad informativa.

Sin duda, esta investigación ofrece, además, resultados en relación con el tipo de contenidos que consiguen despertar mayor engagement político. Así, pudo observarse una relación directa en las 
interacciones sociales cuando el candidato aparecía como protagonista del tuit, circunstancia que se asociaba con un mayor nivel de engagement de los tuits. De este modo, se demuestra cómo la personalización de los tuits resulta ser un recurso efectivo para lograr la adhesión social. Se confirma así nuestra hipótesis de que este tipo de programas con contenido relacionado con la espectacularización de la política (noticias suaves, parodia, tratamiento superficial, entre otros aspectos) puede implicar un mayor grado de engagement en la medida en la que se intensifican algunos encuadres específicos vinculados con los recursos emocionales y la personalización, lo que incrementará el nivel de implicación por parte de la ciudadanía. Estos datos están en la línea de los resultados de investigaciones previas sobre Twitter y politainment (Berrocal, Zamora-Medina y Rebolledo, 2021; Baviera, Peris y Cano-Orón, 2019).

Asimismo, los datos revelaron diferencias significativas en función del tema en cuestión. Cuando estamos ante temas vinculados con cuestiones sociales (soft issues) como desigualdad social o inmigración, el nivel de engagement fue mucho mayor en comparación con cuando se trataba de temas más duros (hard issues) relativos a los políticos o la clase política.

Finalmente, esta investigación ofrece resultados relevantes en la relación entre encuadres y nivel de engagement: los tuits que utilizaron mayoritariamente como marcos interpretativos el encuadre de la emotividad, así como el recurso del conflicto y del humor, lograron despertar mayor implicación del público.

\section{Conclusiones}

El uso del politainment televisivo se utiliza como un recurso de comunicación estratégica mediante el cual los candidatos proyectan su imagen pública y se acercan a nuevos públicos. Por ello, no puede descuidarse sus efectos sobre la audiencia social conectada en las redes sociales. Es precisamente en estos escenarios donde mejor se aprecia la repercusión real de la actuación de los líderes políticos, esto es, a partir de la conversación social generada, en cada caso, con una audiencia social activa, conectada y empoderada.

Esta investigación contribuye al estudio de los posibles efectos de la audiencia social del politainment sobre la generación de engagement ciudadano. El objetivo principal es analizar en qué medida estos espectáculos de entretenimiento contribuyen a mejorar el conocimiento político de cada candidato a través de los ojos de la audiencia social de Twitter y comprobar, asimismo, su idoneidad para el fortalecimiento de la conciencia crítica y democrática.

A partir de un riguroso diseño empírico, los resultados obtenidos en este estudio indican que la conversación social de Twitter ligada al programa de entretenimiento político no favorece la difusión de contenidos políticos constructivos que supongan la mejora del conocimiento político en la ciudadanía. Sin embargo, no por ello dejan de despertar adhesión social; especialmente, cuando estos contenidos se corresponden con tuits personalizados, que enfatizan determinados temas de naturaleza social o que utilizan un enfoque emocional.

Estas aportaciones relativas al estudio del engagement y el politainment, que incluyen también una propuesta metodológica en construcción, no están exentos de algunas limitaciones. Por un lado, se ha tomado como referencia un único programa considerado politainment; por otro lado, el análisis empírico incluye todos los tuits generados en el espacio temporal de un día, el correspondiente a la emisión directa del programa en el que aparecen los candidatos. En futuras investigaciones, sería interesante enriquecer la investigación comparando las diferentes categorías de programas de politainment entre ellas. Asimismo, sería pertinente comparar el tipo de conversación de la audiencia social con las apariciones de políticos en otros programas de corte más informativo y que quedan fuera de la categoría politainment. De este modo, se alcanzaría una visión más completa sobre las 
dinámicas en torno a la conversación que tiene en lugar en la esfera Twitter y comprobar hasta qué punto el conocimiento político está presente de manera general en las redes. Por último y en esta línea, sería conveniente considerar la posibilidad de valorar diferentes tipos de redes sociales de manera comparada con el fin de tener una visión más holística del fenómeno politaiment y la conversación de la audiencia social.

\section{Bibliografía}

Alonso-Muñoz, L. y Casero-Ripollés, A. (2017). Transparencia y monitorización en el entorno digital. Hacia una tipología de las plataformas impulsadas por la ciudadanía. Revista Latina de Comunicación Social, 72, 1.351-1.366. https://doi.org/10.4185/RLCS-2017-1223

Aroldi, P. y Vittadini, N. (2015). Audiences as Socio-Technical Actors: The "Styles" of Social Network Site Users. En F. Zeller, C. Ponte y B. O’Neill. (Eds.). Revitalizing Audience Research: Innovations in European Audience Research, (pp.195-214). New York: Taylor \& Francis.

Ballesteros Herencia, C.A. (2017). Movilización en las campañas electorales a través de las redes sociales. Las elecciones generales españolas de 2015. Paper presentado en the International Association of Media and Communication Research (IAMCR) Conference, Cartagena, Colombia. http://uvadoc.uva.es/handle/10324/26049

Ballesteros Herencia, C.A. (2019). La representación digital del engagement: hacia una percepción del compromiso a través de acciones simbólicas. Revista de Comunicación, 18(1), 215-233. https://doi.org/10.26441/RC18.1-2019-A11

Barlovento. (2019). Análisis televisivo 2019 https://www.barloventocomunicacion.es/audienciasanuales/analisis-televisivo-2019

Baum, M.A. (2003). Soft news and political knowledge: Evidence of absence or absence of evidence? Political Communication, 20, 173-190. https://doi.org/10.1080/10584600390211181

Baviera, T., Peris, À. y Cano-Orón, L. (2019). Political candidates in infotainment programmes and their emotional effects on Twitter: an analysis of the 2015 Spanish general elections precampaign season. Contemporary Social Science, 14(1), 144-156. https://doi.org/10.1080/2158204 $\underline{1.2017 .1367833}$

Berrocal, S., Campos-Domínguez, E. y Redondo, M. (2012). Comunicación Política en Internet: La tendencia al" infoentretenimiento" político en YouTube. Estudios sobre el mensaje periodístico, 18(2), 643-659. https://doi.org/10.5209/rev_ESMP.2012.v18.n2.41037

Berrocal, S., Campos-Domínguez, E. y Redondo, M. (2014). Prosumidores mediáticos en la comunicación política: el «politainment» en YouTube, Comunicar, 21(43), 65-72. https://doi. org/10.3916/c43-2014-06

Berrocal, S., Gil Torres, A. y Campos-Domínguez, E. (2016). El uso de YouTube en las Elecciones al Parlamento Europeo 2014. El caso de España, Comunicación y hombre, (12), 57-72.

Berrocal, S. (Coord.) (2017). Politainment. La política espectáculo en los medios de comunicación. Valencia: Tirant Lo Blanch Humanidades.

Berrocal, S., Zamora-Medina, R. y Rebolledo, M. (2021). Politainment social audience and political engagement: Analysing Twitter conversations in Spain, Catalan Journal of Communication \& Cultural Studies, 13(1), 23-42, https://doi.org/10.1386/cjcs_00037_1 
Boulianne, S. (2009). Does internet use affect engagement? A meta-analysis of research. Political Communication, 26, 193-211. https://doi.org/10.1080/10584600902854363

Boulianne, S. y Theocharis, Y. (2018). Young People, Digital Media, and Engagement: A Meta-Analysis of Research. Social Science Computer Review, 38(2), 111-127. https://doi. org $/ 10.1177 / 0894439318814190$

Boukes M. y Trilling D. (2017). Political relevance in the eye of the beholder: determining the substantiveness of TV shows and political debates with Twitter data. First Monday, 22(4) https:// doi.org/10.5210/fm.v22i4.7031

Buschow, C., Schneider, B. y Ueberheide, S. (2014). Tweeting television: Exploring communication activities on Twitter while watching TV. Communications-The European Journal of Communication Research, 39(2), 129-149. https://doi.org/10.1515/commun-2014-0009

Chae, Y., Lee, S. y Kim, Y. (2019). Meta-analysis of the relationship between Internet use and political participation: examining main and moderating effects. Asian Journal of Communication, 29(1), 35-54. https://doi.org/10.1080/01292986.2018.1499121

Chan-Olmsted, S., Wolter, L.C. y Wang, R. (2017). Toward a Multidimensional Framework of Media Engagement: Conceptualizing Consumer Experience and Connection with Media Content in a Digital Environment. Media Engagement Framework. https://www.mediamanagement.eu/ wp-content/uploads/Chan-Olmsted-Wolter-Wang-2017.-Toward-aMultidimensional-Frameworkof-Media-Engagement.pdf

Cvijikj, I.P. y Michahelles, F. (2013). Online engagement factors on Facebook brand pages. Social Network Analysis and Mining, 3(4), 843-861. https://doi.org/10.1007/s13278-013-0098-8

Diakopoulos, N.A. y Shamma, D.A. (2010). Characterizing debate performance via aggregated twitter sentiment, Proceedings of the SIGCHI Conference on Human Factors in Computing Systems, 1195-1198. https://doi.org/10.1145/1753326.1753504

D'heer, E. y Verdegem, P. (2014). Conversations about the elections on Twitter: Towards a structural understanding of Twitter's relation with the political and the media field. European Journal of Communication, 29(6), 720-734. https://doi.org/10.1177/0267323114544866

Durántez-Stolle, P. y Martínez-Sanz, R. (2019). Politainment in the transmedia construction of the image of politicians. Communication and Society, 32(2), 111-126. https://doi:

10.15581/003.32.2.111-126

Echegaray, L. y Peñafiel, C. (2013). La utilización de las redes sociales como nuevas herramientas aplicadas al análisis de audiencia. Trípodos, 33, 157-172.

Entman, R.M. (1993). Framing: Toward clarification of a fractured paradigm. Journal of Communication, 43 (4), 51-58.

Giglietto, F. y Selva, D. (2014). Second screen and participation: A content analysis on a full season dataset of tweets. Journal of Communication, 64(2), 260-277. https://doi:10.1111/ jcom. 12085

Guo, M. y Chan-Olmsted, S.M. (2015). Predictors of social television viewing: How perceived program, media, and audience characteristics affect social engagement with television programming. Journal of Broadcasting \& Electronic Media, 59(2), 240-258. https://doi.org/10.10 $80 / 08838151.2015 .1029122$ 
Kahne, J. y Bowyer, B. (2018). The political significance of social media activity and social networks. Political Communication, 35(3), 470-493. https://doi.org/10.1080/10584609.2018.1426662

Jiménez, V.M., Barrio, T.V. y Guinovart, E.C. (2017). Los formatos del” politainment” televisivo (The formats of the television "politainment"). En S. Berrocal (Coord.), Politainment: la politica espectáculo en los medios de comunicación (pp. 53-76). Valencia: Tirant lo Blanch.

López-Meri, A., Marcos-García, S. y Casero-Ripollés, A. (2017). What do politicians do on Twitter? Functions and communication strategies in the Spanish electoral campaign of 2016. El profesional de la información, 26(5), 795-804. https://doi.org/10.3145/epi.2017.sep.02

Macnamara, J., Sakinofsky, P. y Beattie, J. (2012). E-electoral engagement: How governments use social media to engage voters. Australian Journal of Political Science, 47 (4), 623-639. https:// doi.org/10.1080/10361146.2012.731491

Marinelli, A. y Andò, R. (2016) Come essere creativi nel nuovo ecosistema mediale. Milano: Fondazione per la Sussidiarietà. http://www.sussidiarieta.net/files/file/rapporto\%20youtube conclusioni.pdf

Moy, P., Xenos, M.A. y Hess, V. K. (2005). Communication and citizenship: Mapping the political effects of infotainment. Mass Communication \& Society, 8(2), 111-131. https://doi.org/10.1207/ $\underline{\mathrm{s} 15327825 \mathrm{mcs} 0802 \_3}$

Muñiz, C. y Ballesteros, C. (2016). Propuesta metodológica para el análisis de la cibercampaña electoral (Methodological proposal for the analysis of the electoral cyber campaign). En J. L. Dader y E. Campos (Coords.), La cibercampaña en Castilla y León: Elecciones Autonómicas, 2015 (pp. 67-82). Valladolid: Ediciones Universidad de Valladolid.

Nieland, J.U. (2008). Politainment. En W. Donsbach (Ed.), The International Encyclopedia of Communication. Blackwell Publishing. https://doi.org/10.1002/9781405186407.wbiecp047

Oliva, M., Pérez-Latorre, Ó. y Besalú, R. (2015). Celebrificación del candidato. Cultura de la fama, marketing electoral y construcción de la imagen pública del político. Arbor, 191 (775); a270. http://dx.doi.org/10.3989/arbor.2015.775n5009

Ortells, S. (2011). La consolidación de los programas de infoentretenimiento en el panorama televisivo español. Fórum de Recerca, 16, 279-291.

Otto, L., Glogger, I. y Boukes, M. (2016). The softening of journalistic political communication: A comprehensive framework model of sensationalism, soft news, infotainment, and tabloidization. Communication Theory, 27(2), 136-155. https://doi.org/10.1111/comt.12102

Oviedo-García, M.A., Muñoz-Expósito, M., Castellanos-Verdugo, M. y Sancho-Mejías, M. (2014). Metric proposal for customer engagement in Facebook. Journal of Research in Interactive Marketing, 8(4), 327-344. https://doi.org/10.1108/JRIM-05-2014-0028

Paine, K.D. (2011). Measure what matters: Online tools for understanding customers, social media, engagement, and key relationships. New Jersey: John Wiley \& Sons.

Pittman, M. y Tefertiller, A. C. (2015). With or without you: Connected viewing and co-viewing Twitter activity for traditional appointment and asynchronous broadcast television models. First Monday, 20(7). https://doi.org/10.5210/fm.v20i7.5935

Sayre, S. y King, C. (2010). Entertainment and society: Influences, impacts, and innovations. New York: Routledge. 
Schultz, D. (2012). Politainment: the ten rules of contemporary politics. USA: Amazon.com.

Semetko, H. y Valkenburg, P. (2000). Framing European politics: a content analysis of press and television news. Journal of communication, 40(2) 93-109. https://doi.org/10.1111/j.1460-2466.2000. tb02843.x

Shamma, D.A., Kennedy, L. y Churchill, E.F. (2009). Tweet the debates: understanding community annotation of uncollected sources, WSM '09: Proceedings of the first SIGMM workshop on Social media, 3-10 https://doi.org/10.1145/1631144.1631148

Skoric, M.M., Zhu, Q., Goh, D. y Pang, N. (2016). Social media and citizen engagement: A meta-analytic review. New Media \& Society, 18(9), 1817-1839. https://doi.org/10.1177/1461444815616221

Teruel Rodríguez, L. (2016). Del Politainment a las redes sociales: ¿Ha servido a los políticos españoles participar del infotainment? Paper presentado en Mediaflows Conference. Valencia, Spain. https://riuma.uma.es/xmlui/bitstream/handle/10630/12574/Art\%C3\%ADculo\%20politainment $\% 20$ valencia $\% 20$ DEF.pdf?sequence $=1$

Tyron, C. (2008). Pop politics: Online parody videos, intertextuality, and political participation. Popular Communication, 6 (4), 209-213. https://doi.org/10.1080/15405700802418537

Towner, T.L. y Dulio, D.A. (2011). An experiment of campaign effects during the YouTube election. New Media \& Society, 4(13), 626-644. https://doi.org/10.1177/1461444810377917

Valerio, G., Herrera-Murillo, D.J., Villanueva-Puente, F., Herrera-Murillo, N. y Rodríguez, M. (2015). Relación entre los formatos de publicación y el engagement digital: estudio de las páginas de Facebook de las universidades mexicanas. RUSC. Universities and Knowledge Society Journal, 12 (1), 50-64. https://doi.org/10.7238/rusc.v12i1.1887 